\title{
Keyhole Revision after Failed Subdural Craniostomy for Chronic Subdural Hematoma
}

\author{
John D. Rolston ${ }^{1}$, Seunggu J. Han ${ }^{2}$, Michael W. McDermott ${ }^{2}$ \\ 1. Department of Neurosurgery, University of California, San Francisco 2. Department of Neurological \\ Surgery, University of California, San Francisco
}

$\square$ Corresponding author: John D. Rolston, rolston2@gmail.com

Disclosures can be found in Additional Information at the end of the article

\section{Abstract}

Introduction: An emerging treatment for chronic subdural hematomas is subdural craniostomy using a twist drill port system. While this system has the advantage of being placed in the intensive care setting, and not the operating room, incomplete hematoma evacuation is not uncommon. We detail a simple surgical technique that can be used for persistent or recurrent collections.

Materials and Methods: For patients with persistent or recurrent chronic subdural hematomas after bedside subdural craniostomy, the patient is taken to the operating room and a "keyhole" burr hole is used that creates a new burr hole in front of the prior twist drill hole. The subdural space is opened such that there is communication between the larger burr hole and craniostomy hole. The bolt for the twist drill system is then re-inserted so that the tip rests just above the dural opening. The anterior burr hole is capped with a titanium burr hole cover with a sector removed.

Results: This method was used in four patients with residual/recurrent subdural hematomas. The mean interval between craniostomy and keyhole revision was 2.8 days (range one to six days). Complications of infection or new acute subdural hematoma occurred in none of the patients. The rate of hematoma recurrence after keyhole revision was $0 \%$. Average operative time for the keyhole procedure was 42.5 minutes (range 28 to $60 \mathrm{~min}$.).

Conclusions: The keyhole method for drainage of residual or recurrent chronic subdural hematomas is an option prior to craniotomy that allows for wider opening of the subdural space for hematoma irrigation and removal, while maintaining postoperative extradural suction drainage.

Received 05/18/2013

Review began 05/19/2013

Published 07/02/2013

C Copyright 2013

Rolston et al. This is an open access article distributed under the terms of the Creative Commons Attribution License CC-BY 3.0., which permits unrestricted use, distribution, and reproduction in any medium, provided the original author and source are credited.
Categories: Neurosurgery

Keywords: keyhole, burr hole, subdural hematoma, subdural drainage

\section{Introduction}

Chronic subdural hematoma (cSDH) is an unfortunately common disorder disproportionately affecting the elderly, with an incidence of 58 per 100,000 individuals yearly over the age of 65 [1]. This corresponds to roughly 24,000 new cases each year in the United States alone, where over $13 \%$ of the population is in this age group, a number greater than that of newly diagnosed primary brain tumors $(18,000)[2]$. 
Until the 1960s, cSDH was primarily treated with craniotomy and hematoma evacuation. However, in 1964, Svien et al. published a series of patients assigned to either craniotomy or burr hole drainage (BHD), and showed that recurrence and functional outcomes were better in patients treated with burr hole craniostomy [3]. Since then, several meta-analyses have supported this approach, and BHD remains the most frequently used treatment. The use of burr holes as the prime treatment for uncomplicated cSDH is also supported by the systematic review of Weigel et al. [4] and the decision analysis of Lega et al. [5].

Several studies have been devoted to the refinement of BHD for cSDH. Of note, Santarius et al. published class I evidence that the presence of a subdural drain left in place postoperatively reduced the recurrence of cSDH and improved patient outcomes [6]. Further research has compared single vs. double burr hole placement, and several studies, e.g., Taussky et al. [7] and Han et al. [8], showed higher recurrence rates with one burr hole vs. two.

The recurrence rate of cSDH remains high at 9.3\%, despite the use of optimal BHD techniques like those of Santarius et al. [6] (multiple burr holes with an indwelling subdural drain). This rate is nearly tripled to $24 \%$ without the use of drains.

Twist drill craniostomy is an alternative method of cSDH drainage with the advantage of being conducted at bedside, typically in an intensive care setting. This has the potential of lower costs (conscious sedation in the ICU vs. mobilization of an operating room and general anesthesia) and faster time to intervention. The Subdural Evacuating Port System (SEPS ${ }^{\mathrm{TM}}$ ) by Medtronic is one of the most frequently used examples of this idea. Twist drill craniostomy appears to have morbidity similar to that of BHD, with complications reported in $0-18 \%$ of patients with twist drill procedures $[9,10,11]$ vs. $0-25 \%$ for $\mathrm{BHD}[12,13]$. Yet the rates of recurrence are empirically higher with bedside procedures: $25.9 \%$ with SEPS vs. $14.8 \%$ with BHD in the study of Rughani et al. [14]; 18\% (twist drill) vs. 8\% (BHD) requiring reoperation/drainage in the study of Horn et al. [9]; and, similarly, 17\% (twist drill) vs. $0 \%$ (BHD and craniotomy) rates of inadequate SDH drainage in the study of Safain et al. [15]. Many of these differences are not reported as statistically significant, but this is possibly due to the low power of the studies. And at least one study, Gokmen et al. [16], showed no difference in recurrence rates between the two techniques. Given the potential benefits of bedside procedures, larger comparison studies between twist drill craniostomy and BHD are definitely needed.

The reasons behind the apparent increased number of reoperations after twist drill craniostomy are unknown. One possibility is that, given the relative scale of the procedures, practitioners are more likely to see BHD as a second-line option for patients who already underwent twist-drill procedures. This is in comparison to patients who underwent BHD upfront, in which case the next "larger" operation would be a full craniotomy. There is also a possible preference for physicians to use twist-drill procedures as temporizing measures until OR time can be safely obtained, which would also spuriously elevate the number of reoperations in that cohort compared to BHD.

Regardless of the reasons, given the high rates of recurrence and need for reoperation after twist drill craniostomy (18-26\%), attention must be paid to the techniques involved with revising prior bedside craniostomies. Here, we propose revision with a "keyhole" burr hole, allowing expanded drainage of the recurrent cSDH and continued maintenance of the subdural drain. Using a simple expansion of the prior craniostomy, it is less invasive than craniotomy or additional burr holes, and thereby potentially less morbid and more economical.

\section{Technical Report}

Patients with persistent chronic subdural hematomas after bedside craniostomy were identified 
by persistence of clinical symptoms, and confirmed by computed tomography (CT). After informed consent of the patient or caregiver, these patients were taken to the operating room for keyhole revision. The area surrounding the prior craniostomy was first sterilized with a mixture of $2 \%$ chlorhexidine and 70\% isopropyl alcohol (ChloraPrep; CareFusion, San Diego, CA, USA). The existing subdural port and drain were removed. The area was then prepped again and draped in typical sterile fashion.

The prior skin incision was extended and the opening maintained with a surgical retractor. The periosteum was removed with a periosteal elevator and electrocautery as appropriate. A new burr hole was then drilled adjacent to the prior twist drill craniostomy, incorporating the prior craniostomy partially on its outer diameter (Figures $1 B, 1 C$ ). The two holes were therefore contiguous. The new burr hole was roughly three times the diameter of the twist-drill craniostomy. At this point, the dura was exposed using a combination of curette and rongeurs. The exposed dura was then incised using a \#15 blade (Figure 1D). Bipolar electrocautery was used to stop and dural bleeding.

At this point, the exposed subdural space was irrigated with normal saline to reduce the volume of the recurrent subdural hematoma. This was repeated until only clear saline returned after each delivery of irrigation fluid.

A new, sterile bolt was then inserted into the prior twist drill craniostomy until its tip approximated the subdural opening (Figure $1 E$ ). The larger burr hole was then capped with a titanium burr hole cover with one sector removed (Universal Neuro II plating system; Stryker, Kalamazoo, MI, USA). The removed sector provides space for the subdural port to exit (Figure $1 F)$. 


\section{Cureus}

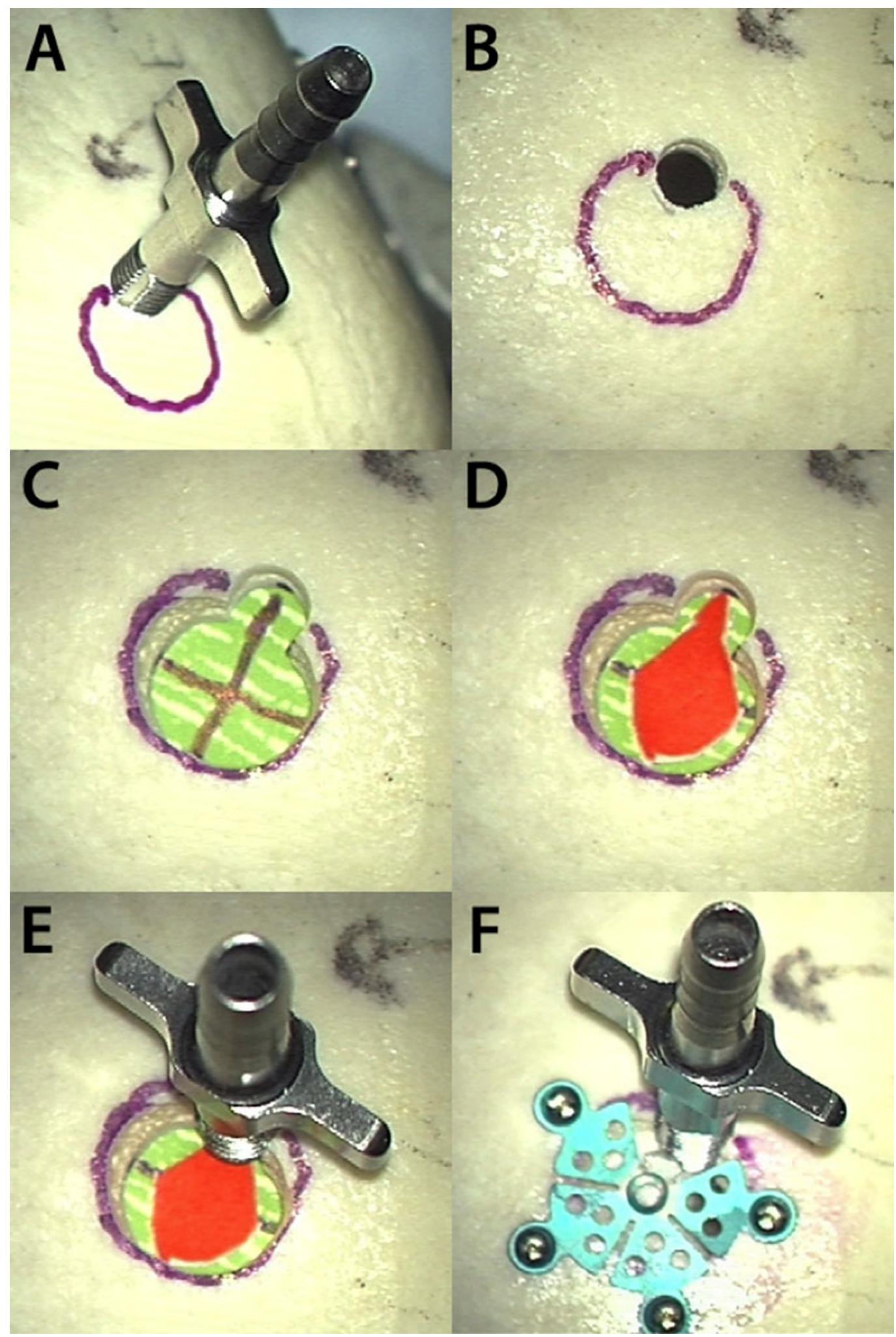

FIGURE 1: Overview of keyhole revision on cadaveric skull.

(A) Preexisting subdural port with new burr hole outlined. (B) Subdural port removed. (C) Dural incision (dura represented by green). (D) Dura after incision (subdural contents represented by red filling). (E) Subdural port replaced. (F) Burr hole cover attached, with sector removed to accommodate subdural port. 


\section{Cureus}

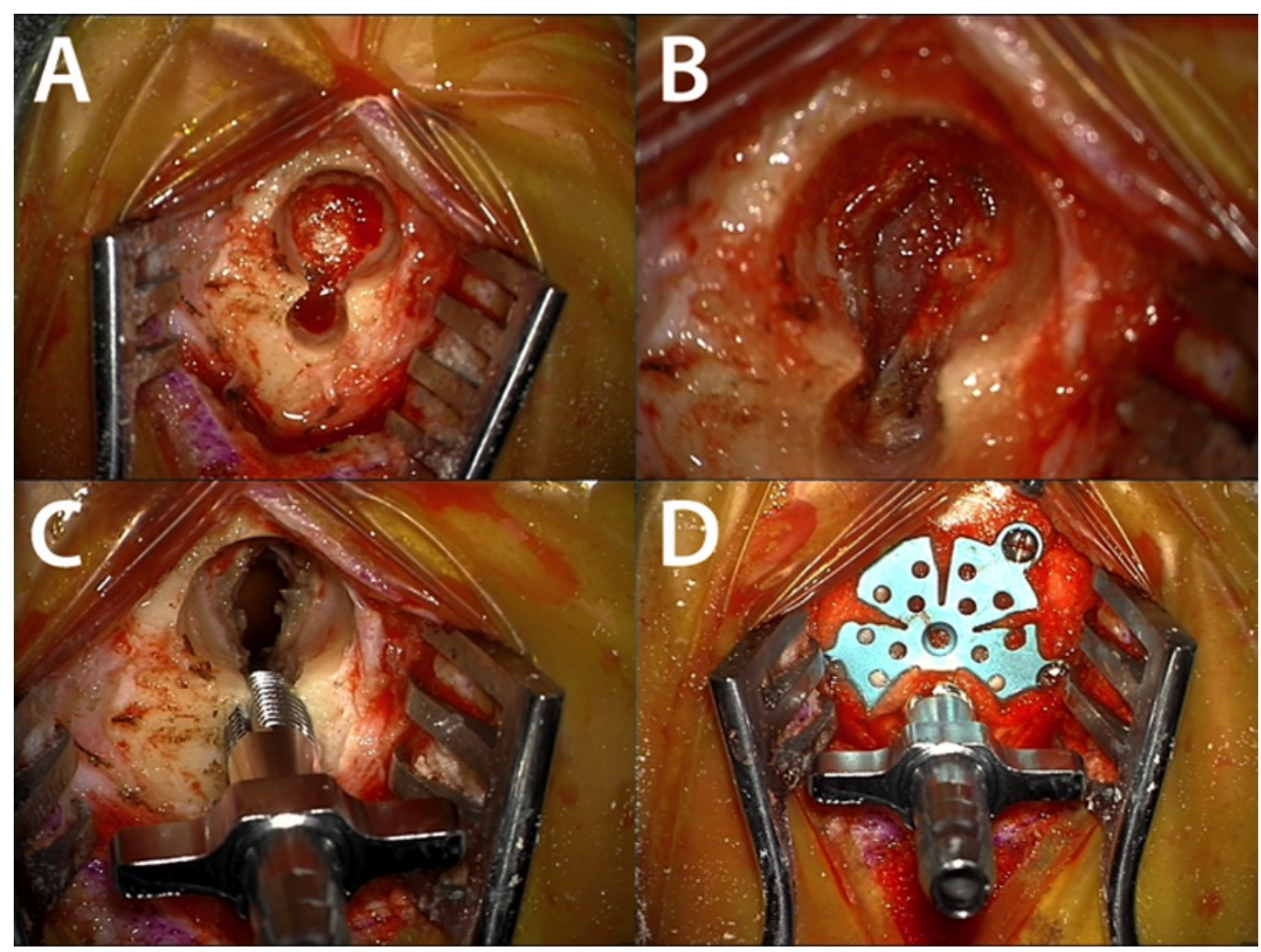

FIGURE 2: Intraoperative photos of keyhole revision.

(A) New burr hole drilled contiguous with prior twist drill craniostomy. (B) Dural incision revealed cSDH. (C) After copious irrigation, a new subdural port is implanted. (D) A burr hole cover with one sector removed is secured over the new burr hole.

The incision was then closed in layers in the standard fashion. At the end of the procedure, the subdural port was then reattached to a sterile suction tube connected to bulb suction.

\section{Results}

Four patients were found to have recurrent cSDH and treated with keyhole revisions. The interval between craniostomy and keyhole revision ranged from 1 to 6 days (median 2 days). Complications of infection or new acute subdural hematoma occurred in no patients. The recurrence rate after keyhole revision, as determined by CT scan, was $0 \%$. Operative time for the keyhole procedure was 42.5 minutes (range 28 to $60 \mathrm{~min}$ ).

\section{Discussion}

Twist drill craniostomy for the evacuation of cSDH has the important advantage of being a bedside procedure with good results $[1,5,9,14]$. This translates to potentials savings in both cost and time, as compared to burr hole drainage in the operating room. However, as with all methods of cSDH evacuation, there are failures. In $18-26 \%$ of cases, the subdural collection recurs $[1,5,9,14]$. These patients require revision. and, unfortunately, even a handful of reoperations can largely negate the economic benefits of bedside procedures.

Above, we describe a novel technique of revising twist-drill craniostomies using a subdural evacuation port system. This method maintains the important aspect of subdural drainage, which has previously been shown as superior to evacuation without continued drainage in a randomized clinical trial [6]. After patients were identified with failed bedside drainage, they 
were taken to the operating room for a "keyhole" revision of their prior twist drill craniostomy. A new burr hole was created in continuity with the pre-existing craniostomy, subdural fluid was evacuated, and the prior subdural port was reimplanted to provide continued postop drainage (see Methods above).

Keyhole revision has been performed in 4 patients at our institution so far. There have been no complications intraoperatively. Recurrence of hematoma after revision occurred in $0 \%$ of cases, comparing favorably to other methods of cSDH evacuation. Mean operative time was 42.5 minutes, which again compares favorably to traditional methods of hematoma evacuation.

\section{Conclusions}

Though recurrent cSDH remains an important problem, it is our hope that this simple method provides a useful tool for neurosurgeons faced with failed twist-drill craniostomies. It is also possible to consider doing such an operation at bedside, provided the procedure described above was appropriately modified. While this is a preliminary report describing the technique, we believe that more thorough study of this method's efficacy, along with the efficacy of other revision methods, is critically needed. Newly diagnosed subdural hematomas afflict nearly 24,000 individuals yearly in the US alone, more than are diagnosed with primary brain tumors. Any innovations that can improve the outcome of these patients will be valuable contributions to our field, both improving patient outcomes and lowering the cost for medical care.

\section{Additional Information \\ Disclosures}

Human subjects: Consent was obtained by all participants in this study. Concerning the use of human subjects: All patients provided written and verbal informed consent before participating in the described surgical procedures. Procedures were carried out according to institutional guidelines. . Animal subjects: All authors have confirmed that this study did not involve animal subjects or tissue. Conflicts of interest: In compliance with the ICMJE uniform disclosure form, all authors declare the following: Payment/services info: All authors have declared that no financial support was received from any organization for the submitted work. Financial relationships: All authors have declared that they have no financial relationships at present or within the previous three years with any organizations that might have an interest in the submitted work. Other relationships: All authors have declared that there are no other relationships or activities that could appear to have influenced the submitted work.

\section{References}

1. Kudo H, Kuwamura K, Izawa I, Sawa H, Tamaki N: Chronic subdural hematoma in elderly people: present status on Awaji Island and epidemiological prospect. Neurol Med Chir (Tokyo). 1992, 32:207-209.

2. Chandana SR, Movva S, Arora M, Singh T: Primary brain tumors in adults . Am Fam Physician. 2008, 77:1423-1430.

3. Svien HJ, Gelety JE: On the Surgical Management of Encapsulated Subdural Hematoma. A Comparison of the Results of Membranectomy and Simple Evacuation. J Neurosurg. 1964, 21:172-177.

4. Weigel R, Schmiedek P, Krauss JK: Outcome of contemporary surgery for chronic subdural haematoma: Evidence based review. J Neurol Neurosurg Psychiatry. 2003, 74:937-943.

5. Lega BC, Danish SF, Malhotra NR, Sonnad SS, Stein SC: Choosing the best operation for chronic subdural hematoma: a decision analysis. J Neurosurg . 2010, 113:615-621.

6. Santarius T, Kirkpatrick PJ, Ganesan D, Chia HL, Jalloh I, Smielewski P, et al.: Use of drains versus no drains after burr-hole evacuation of chronic subdural haematoma: A randomised controlled trial. Lancet. 2009, 374:1067-1073.

7. Taussky P, Fandino J, Landolt H: Number of burr holes as independent predictor of 
postoperative recurrence in chronic subdural haematoma. Br J Neurosurg . 2008, 22:279-282.

8. Han HJ, Park CW, Kim EY, Yoo CJ, Kim YB, Kim WK: One vs. Two Burr Hole Craniostomy in Surgical Treatment of Chronic Subdural Hematoma. J Korean Neurosurg Soc . 2009, 46:87-92.

9. Horn EM, Feiz-Erfan I, Bristol RE, Spetzler RF, Harrington TR: Bedside twist drill craniostomy for chronic subdural hematoma: a comparative study. Surg Neurol. 2006, 65:150-153.

10. Santos-Ditto RA, Santos-Franco JA, Pinos-Gavilanes MW, Mora-Benitez H, Saavedra T, Martinez-Gonzales V: Management of chronic subdural hematoma with twist-drill craniostomy. Report of 213 patients. Gac Med Mex . 2007, 143:203-208.

11. Smely C, Madlinger A, Scheremet R: Chronic subdural haematoma--a comparison of two different treatment modalities. Acta Neurochir (Wien). 1997, 139:818-825.

12. Ducruet AF, Grobelny BT, Zacharia BE, Hickman ZL, DeRosa PL, Anderson K, et al. : The surgical management of chronic subdural hematoma. Neurosurg Rev. 2012, 35:155-169.

13. Tanikawa M, Mase M, Yamada K, Yamashita N, Matsumoto T, Banno T, et al.: Surgical treatment of chronic subdural hematoma based on intrahematomal membrane structure on MRI. Acta Neurochir (Wien). 2001, 143:613-618.

14. Rughani AI, Lin C, Dumont TM, Penar PL, Horgan MA Tranmer BI: A case-comparison study of the subdural evacuating port system in treating chronic subdural hematomas. J Neurosurg. 2010, 113:609-614.

15. Safain M, Roguski M, Antoniou A, Schirmer CS, Malek AM, Riesenburger R: A single center's experience with the bedside subdural evacuating port system: a useful alternative to traditional methods for chronic subdural hematoma evacuation. J Neurosurg . 2013 , 118:694700.

16. Gokmen M, Sucu HK, Ergin A, Gokmen A, Bezircio Lu H: Randomized comparative study of burr-hole craniostomy versus twist drill craniostomy; surgical management of unilateral hemispheric chronic subdural hematomas. Zentralbl Neurochir. 2008, 69:129-133. 